\title{
Two different coercivity lattices in Co/Pd multilayers generated by single- pulse direct laser interference lithography
}

\author{
Philipp M. Leufke, ${ }^{1, \text { aj }}$ Stephen Riedel, ${ }^{1}$ Min-Sang Lee, ${ }^{3}$ Jie Li ${ }^{3}$ Hartmut Rohrmann, ${ }^{2}$ \\ Thomas Eimüller, ${ }^{3, \text { c) }}$ Paul Leiderer, ${ }^{1}$ Johannes Boneberg, ${ }^{1}$ Günter Schatz, ${ }^{1}$ and \\ Manfred Albrecht ${ }^{1, b)}$ \\ ${ }^{1}$ Department of Physics, University of Konstanz, 78457 Konstanz, Germany \\ ${ }^{2}$ OC Oerlikon Balzers $A G, 9496$ Balzers, Liechtenstein \\ ${ }^{3}$ Junior Research Group Magnetic Microscopy, Ruhr-University of Bochum, 44801 Bochum, Germany
}

\begin{abstract}
We report on magnetic structuring of $\mathrm{Co} / \mathrm{Pd}$ multilayer films with strong perpendicular magnetic anisotropy by single-pulse direct laser interference lithography technique. Multibeam laser interference generates patterns of various types. The intense laser irradiation at interference maxima causes chemical intermixing at $\mathrm{Co} / \mathrm{Pd}$ interfaces, leading to local changes in magnetic properties such as the creation of pinning centers and the reduction in the strength of magnetic anisotropy. We use magnetic force microscopy and Kerr microscopy to study the magnetization reversal processes in the patterned samples and find that the structures show three distinctly different behaviors depending on the intensity of the laser used for irradiation.
\end{abstract}

\section{INTRODUCTION}

Increasing demands for high-density information storage media have led to the development of many novel nanofabrication techniques. They include lithographical methods using electron beam ${ }^{1,2}$ or focused ion beam, 3,4 nanoimprinting, ${ }^{2,5}$ ion-beam milling/mixing, ${ }^{6,7}$ various methods making use of self-assembly processes, ${ }^{8-10}$ and many others, where one technique is sometimes applied in combination with another. Among all these methods the direct laser interference lithography ${ }^{1-18}$ (DLIL) offers the advantage that a large area of a metal film can be homogeneously patterned directly without the need of resists, templates, prepatterns, or large high-cost facilities.

Alongside this development in fabrication of small-size lateral magnetic structures, there have also been a lot of research activities aimed to understand and take technological advantage of the properties of magnetic heterostructures. ${ }^{19}$ In particular, multilayer materials with perpendicular magnetic anisotropy (PMA) are of great technological interest because they promise high information density for magnetoelectronic devices. ${ }^{20-23}$ Especially, Co/Pd multilayers, which are the samples studied in the present work, are interesting not only from the technological point of view but also with respect to fundamental research. Although they have been investigated for more than 20 years, there are still debates about the microscopic origin of the PMA in Co/Pd multilayers. The naive assumption of the symmetry breaking at $\mathrm{Co} / \mathrm{Pd}$ interfaces being the only cause for the PMA has been shown to be obsolete over the past years. ${ }^{24-27}$ One important factor influ-

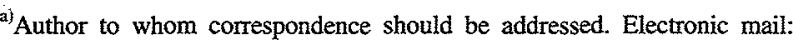
philipp.leufke@int.fzk.de. Present address: Institute of Nanotechnology, Karlsruhe Research Centre, 76021 Karlsruhe, Germany.

${ }^{b}$ Present address: Institute of Physics, Chemnitz University of Technology, 09107 Chemnitz, Germany.

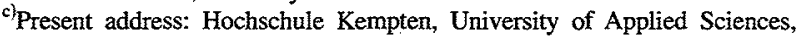
Bahnhofstr. 61, 87435 Kempten, Germany.
}

encing the strength of the anisotropy is found to be of magnetoelastic nature, ${ }^{28}$ where the strain at the interfaces can originate from many different sources such as layer deposition process, ${ }^{27}$ lattice mismatch, ${ }^{29}$ the difference in thermal expansion coefficients of $\mathrm{Co}$ and $\mathrm{Pd}$, and from the magnetoelasticity of CoPd alloys under stress at the interfaces. ${ }^{30,31}$ Other reports show that the strong hybridization between $\mathrm{Co}$ $3 d$ and Pd $4 d$ states at the interface directly correlates with the magnitude of the PMA. ${ }^{28,32,33}$

In any case, there is no doubt that the magnetic properties of $\mathrm{Co} / \mathrm{Pd}$, such as the anisotropy, strongly depend on its interfacial properties, and therefore, it must be possible to tune the magnetic properties of $\mathrm{Co} / \mathrm{Pd}$ by modifying the interfacial structure and chemistry. It has already been demonstrated that both the structure and chemistry at multilayer interfaces can be modified locally in a controlled way using either an ion beam ${ }^{6,34-36}$ or a laser beam. ${ }^{18}$ In the present work, the latter is used in form of single-pulse DLIL to magnetically structure Co/Pd multilayer systems with strong PMA. It will be shown how their magnetic properties and magnetization reversal processes can be tuned locally by varying the laser intensity.

Section II deals with the preparation of the Co/Pd samples, as well as the setup and parameters used for the single-pulse DLIL process. In addition, the as-grown Co/Pd sample is characterized by superconducting quantum interference device (SQUTD) magnetometry, atomic force microscopy (AFM), magnetic force microscopy (MFM), and Kerr microscopy. These results are used as reference data to be compared to the ones obtained for the structured samples in Sec. III where the measurement results are presented and discussed.

\section{SAMPLE PREPARATION AND CHARACTERIZATION}

Figure 1(a) shows the detailed structure of the $\mathrm{Co} / \mathrm{Pd}$ multilayer samples. These multilayers were fabricated in the 
b)
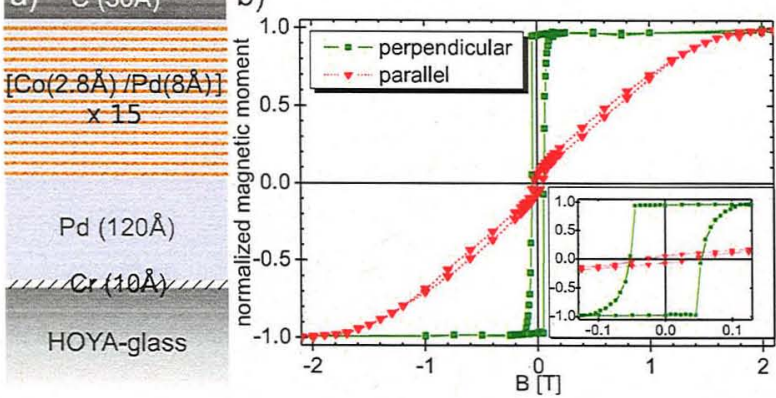

FIG. 1. (Color online) (a) The composition and structure of the Co/Pd multilayer system. (b) Hysteresis loops measured for the easy axis (solid/ green; perpendicular to the sample plane) and for the hard axis (dotted/red; parallel to the sample plane) by SQUID magnetometry. In the inset a magnified view of the central part of the loops is shown to facilitate the estimation and comparison of the coercivities.

magnetron sputtering system RACETRACK by OC Oerlikon Corporation in Balzers, Liechtenstein. The glass substrate was supplied by HOYA Corporation and is of the kind that has a very flat and smooth surface and is used in hard disk drives. The thin chromium layer works as adhesion promoter between the glass substrate and the 120 - $\AA$-thick palladium seed-layer, on top of which 15 repetitions of the $\mathrm{Co} / \mathrm{Pd}$ bilayer are deposited. For protection against oxidation and mechanical influences from outside, the multilayer is capped with a 50 - $\AA$-thick layer of diamondlike carbon. The bilayer composition of $\mathrm{Co}(2.8 \AA) / \mathrm{Pd}(8 \AA)$ ensures the strong PMA $^{25,27}$ as confirmed by the hysteresis loops in Fig. 1(b) measured by SQUID magnetometry.

The MFM image in Fig. 2(a) shows that the as-grown sample exhibits an irregular stripe-domain pattern at remanence, which is characteristic of ferromagnetic multilayer systems with PMA. ${ }^{37-40}$ The magnetization reversal process was studied using Kerr microscopy, which revealed that the reversal occurs by domain wall propagation as shown in Fig.

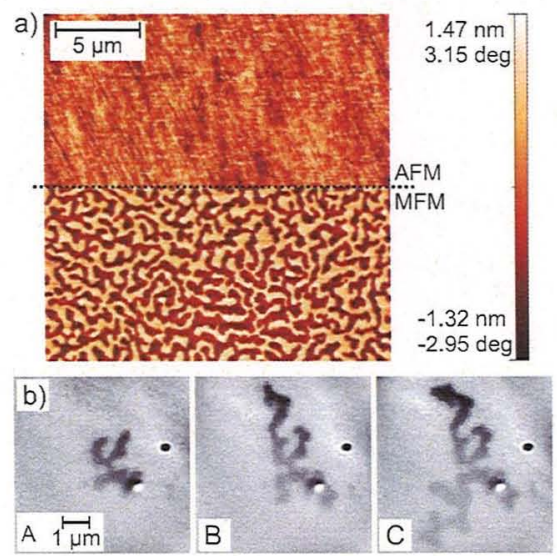

FIG. 2. (Color online) (a) The topography and domain structure of the as-grown sample imaged by AFM and MFM, respectively; the scale bar on the right of the AFM and MFM image represents the height contrast scale for AFM and magnetic contrast scale (phase shift) for MFM; the rmsroughness measured by AFM was approximately $1 \mathrm{~nm}$. (b) The growth of a domain by domain wall propagation recorded in three consecutive Kerr microscopy images at an external reversal field of $-66.8 \mathrm{mT}$. 2(b). If the sample is irradiated by a laser, the domain pattern at remanence as well as the reversal characteristics will be changed due to the alteration of magnetic properties resulting from the heat-induced chemical mixing at the interfaces. Schuppler et al. ${ }^{10}$ demonstrated that such alteration can be realized for $\mathrm{Co} / \mathrm{Pt}$ multilayers in a controlled way using a single-pulse laser. It was concluded that the chemical mixing at the interfaces causes an enhancement in saturation magnetization and a reduction in the strength of PMA. For Co/Pd multilayers, however, we found out in experiments with homogeneously irradiated samples that the irradiation by a single laser pulse leads to a small reduction (approximately $5 \%$ ) of saturation magnetization and a significant reduction in the strength of PMA (approximately 20\%-30\%). ${ }^{41}$ Making use of this knowledge we apply single-pulse DLIL to fabricate lateral structures on $\mathrm{Co} / \mathrm{Pd}$ multilayers and study their altered magnetic properties and reversal processes. As pointed out earlier, the main advantage of this structuring method is that it involves only one single processing step, which is the direct laser irradiation of the metal film. In fact, this requires a very short period of time $(\sim 10 \mathrm{~ns})$ because exposure to only one single laser pulse is sufficient for the fabrication.

DLIL was first introduced by Polushkin and co-workers. ${ }^{11,42}$ Later, Aktag et al. ${ }^{18}$ applied an advanced four-beam interference patterning apparatus, which was used to structure $\mathrm{Co} / \mathrm{Pt}$ multilayer systems. We use a similar setup with a frequency-doubled $Q$-switched neodymium-doped yttrium aluminum garnet (Nd:YAG) laser, providing $10 \mathrm{~ns}$ pulses with a single-pulse energy of $200 \mathrm{~mJ}$ at the wavelength of $532 \mathrm{~nm}$. An injection seeder ensures a long coherence length. Passing through beam splitters and reflecting off several mirrors, the laser beam is split into many beams with approximately equal intensities that are directed onto the sample surface where they interfere with each other. With such a setup, two-dimensional patterns of various types and shapes can be generated by changing the number of interfering beams, the angle of incidence, and the polarization of each beam. For the fabrication of the structures studied here only two- and three-beam interference patterns have been used.

\section{RESULTS AND DISCUSSION}

Figures 3(a)-3(d) show the simulated two-beam and three-beam interference patterns and the AFM images of the corresponding real structures on the $\mathrm{Co} / \mathrm{Pd}$ multilayer samples. As the AFM images in Figs. 3(b) and 3(d) show, the localized high intensity at the interference maxima has produced protrusions on the sample surface, which will hereafter be referred to as "hillocks," whereas the surrounding lower lying regions will be referred to as "valleys." Figures 3(b) and 3(d) show regions that were located in the center of the irradiated area, where the laser intensity was highest and therefore the height contrast is very distinct. We observed that the height contrast between the hillocks and the valleys gradually decreases from the center toward the rim. This is due to the Gaussian intensity profile of the incident laser beams used during the structuring process. For the peripheral 


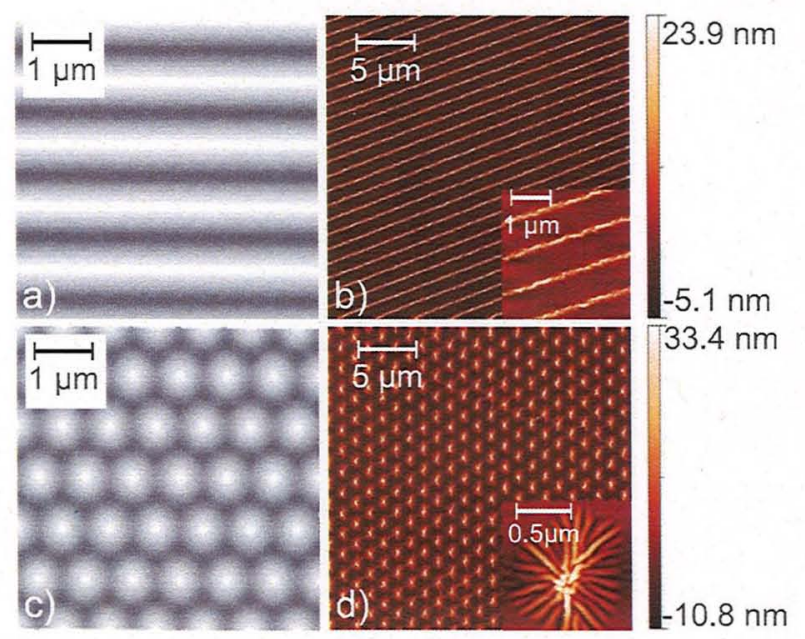

FIG. 3. (Color online) [(a) and (c)] Simulated two-beam and three-beam laser interference pattern, respectively. [(b) and (d)] Topography images (AFM) of the Co/Pd multilayer system structured with two-beam laser interference and with three-beam laser interference, respectively.

regions, the topographical changes in the sample surface were much less pronounced, down to and below the detection limit of AFM. However, it will be shown below that the magnetic patterning of the sample has still been possible even for regions where the topographical changes can hardly be seen.

Using focused polar magneto-optic Kerr effect (MOKE), MFM, and Kerr microscopy we studied the magnetization reversal processes in regions that had been exposed to different laser intensities during the patterning process. Here, we first show the results from the measurements made for an area that was located in the central part of the Gaussian beam profile and therefore irradiated at the highest laser intensity (see Figs. 4 and 5). We refer to this area as "region I." Measuring the hysteresis loop by focused polar MOKE we found that the coercivity was greatly enhanced compared to the one of the as grown sample [see Fig. 4(a))].

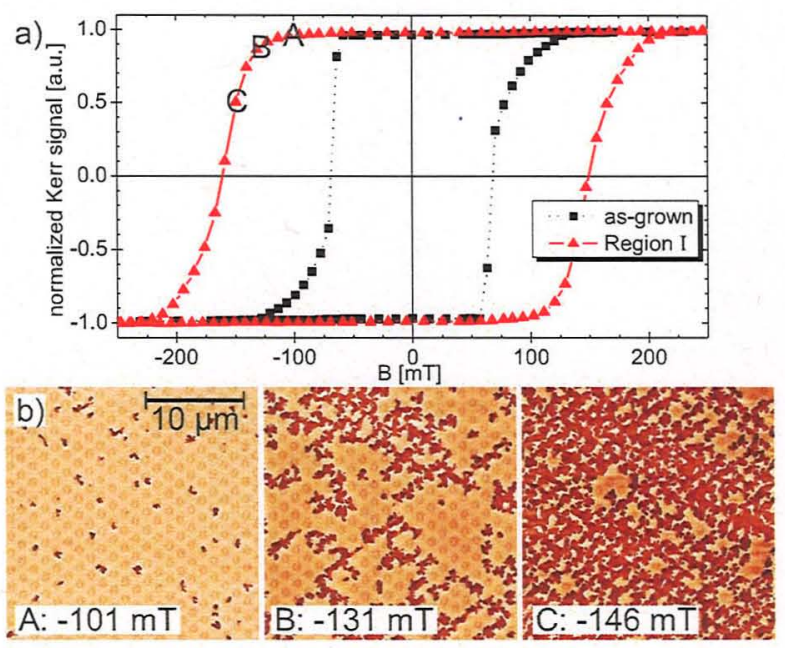

FIG. 4. (Color online) (a) Focused polar MOKE hysteresis loop and (b) MFM micrographs recorded at different remanent magnetizations $A-C$. The measurements were carried out in a region irradiated at high laser intensity (region I).

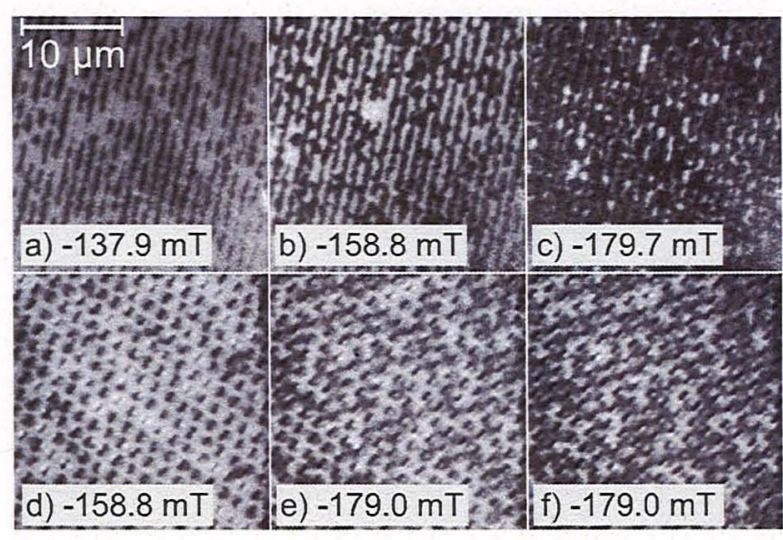

FIG. 5. Kerr microscopy images of magnetization reversal in region I. [(a)(c)] Sample patterned with two-beam interference. [(d)-(f)] Sample patterned with three-beam interference.

Figure 4(b) shows MFM images recorded for different remanent states $A, B$, and $C$. Before monitoring each remanent state by MFM, the sample was first saturated in order to have a well-defined initial condition. Then a magnetic field of a given strength was applied in the direction opposite to the previous saturation field. Our interpretation of the these images is that the magnetization reversal is dominated by the nucleation of small domains separated and isolated from one another in great contrast to the case of the as-grown sample shown in Fig. 2(b).

The MFM offers high resolution magnetic imaging. It probes, however, only static remanent states. To obtain complementary results, we also applied Kerr microscopy and recorded in-field live images of magnetization reversal processes. For fast image acquisition a 1.4 megapixel chargecoupled device camera providing a maximum frame rate of $30 \mathrm{fps}$ was used. The Kerr microscopy images in Fig. 5 confirm the interpretation of the MFM images and, in addition, show that the nucleation always starts on the hillocks or in the direct vicinity of the hillocks first, i.e., the places that were exposed to the interference maxima. The switching of the valleys follows hereafter.

The areas irradiated at lower laser intensities (region II) exhibit a quite different switching behavior. The measurement results shown in Figs. 6 and 7 have been obtained for areas where topographical changes on the sample surface are minute. First, the focused polar MOKE hysteresis loops in Fig. 6(a) show that also here, the coercivity has increased with respect to the as-grown sample, however, to a much lower extent than in region I [cf. Fig. 4(a)]. Second, the hysteresis curve measured for region II in Fig. 6(a) has abrupt changes in its path, in contrast to the one shown in Fig. 4(a), which is rounder and smoother. This is due to the fact that the magnetization reversal in region II is dominated by rapid domain wall propagation, which starts abruptly when the external field exceeds a certain threshold, whereas in region I the number of small isolated domains increases rather gradually by many successive nucleation processes.

The first abrupt change in MOKE signal in Fig. 6(a) is found at the nucleation field of approximately $-80 \mathrm{mT}$. This initiates the domain wall propagation along the valleys. Fig- 


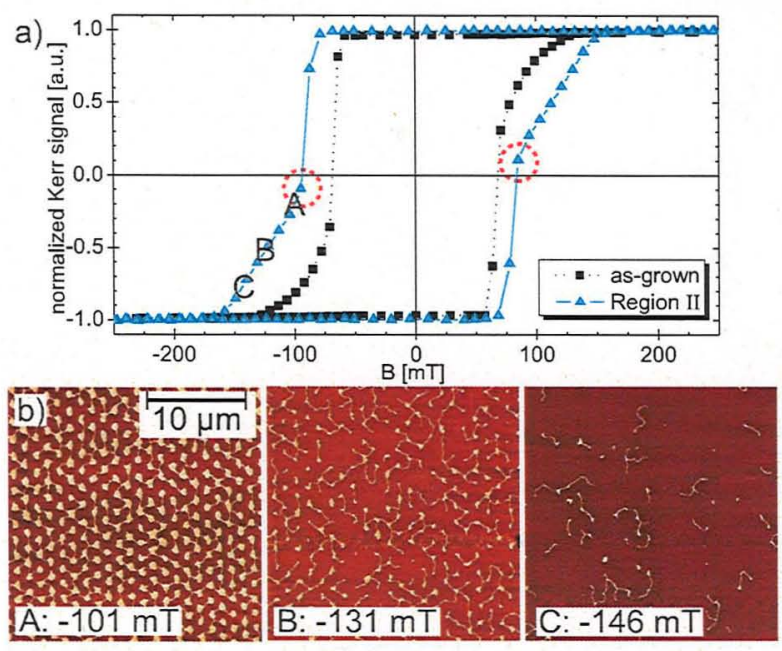

FIG. 6. (Color online) (a) Focused polar MOKE hysteresis loop and (b) MFM micrographs recorded at different remanent magnetizations. The measurements were carried out in a region irradiated at medium laser intensity (region II). The dotted red circles mark the field threshold above which the hillocks start to switch.

ures 7(a)-7(f) show that the hillocks in region II obstruct the domain wall propagation, forcing the domains to grow along the valleys. Therefore, compared to the magnetization reversal in the as-grown sample, shown in Fig. 2(b), the domains in region II grow in a more orderly manner. At approximately $-100 \mathrm{mT}$ another abrupt change appears in the hysteresis. The kink highlighted with a dotted red circle in Fig. 6(a) results from the fact that the hillocks in region II are harder to switch than the valleys. Note that the switching order in region II is opposite to the case of region I. To switch the hillocks, the magnitude of the external reversal field has to be further increased above another threshold of approximately $100 \mathrm{mT}$ at which the domain walls start to propagate toward the hillocks, making the minority domains in the hillocks shrink and vanish. The MFM images A-C in Fig. 6(b) illustrate this process.

There is another area that we refer to as "region III." This area was located at the outer rim of the Gaussian beam

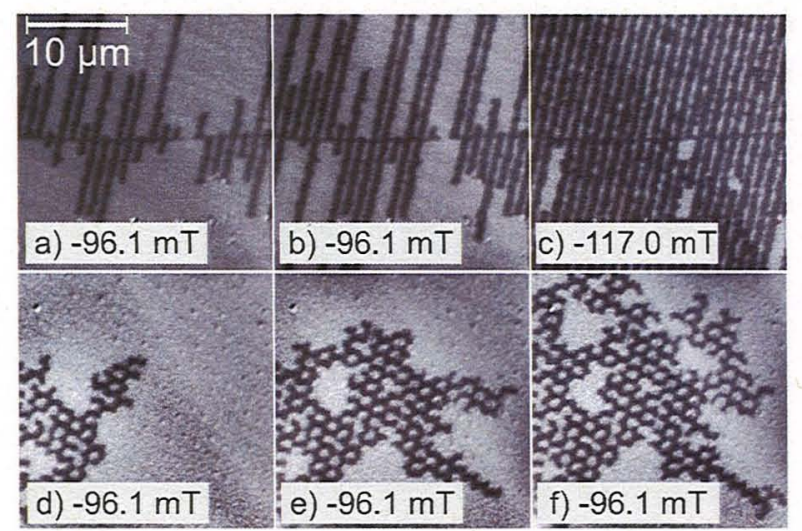

FIG. 7. Kerr microscopy images of magnetization reversal in region II. [(a)-(c)] Sample prepared with two-beam interference. [(d)-(f)] Sample patterned with three-beam interference. The thin horizontal line in (a)-(c) is a scratch on the surface, which acts as a nucleation site.

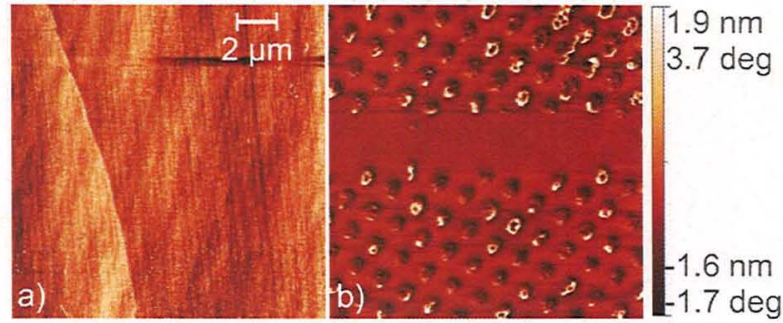

FIG. 8. (Color online) (a) AFM and (b) MFM micrographs of region III (region irradiated at very low intensity) on a sample patterned with threebeam interference. The flattened area in (b) was scanned twice and shows the magnetic contrast recorded at the second run. The first scan started from the middle line and ended approximately $2 \mu \mathrm{m}$ above.

profile and therefore exposed to very low yet finite laser intensity. We actually studied this region by MFM immediately after the patterning process without applying any external magnetic field to the sample. Figures 8 (a) and 8(b) show that the laser irradiation did not cause any resolvable topographical changes to the sample surface at all, yet there is a magnetic pattern observable by MFM. This magnetic pattern disappears, however, already under the influence of the stray field of an MFM tip, which was observed by scanning the same area twice with the MFM tip. The result is the flattened area in the middle of Fig. 8(b). The magnetic contrast of the twice scanned area does not show any regular pattern, whereas a pattern such as the ones observed in neighboring areas was clearly visible at the first run. This indicates that the laser intensity for region III was so weak that the irradiation did not cause any permanent changes to the magnetic structure of the sample but only thermal excitation of the magnetic moments. We could confirm by MFM and Kerr microscopy that the periodic magnetic pattern shown in Fig. 8 disappeared after an external field had been applied to the sample.

To explain the reason for the distinctly different switching behaviors in the three regions, one has to think about the process of structural alteration of the sample by laser irradiation. The laser-induced hillock formation observed by AFM in Figs. 3(b) and 3(d) indicates that the intense laser heating of the sample at the interference maxima caused melt flow from the surrounding colder areas into the hot centers of interference maxima. Supposedly, both the surface tension gradient and the chemical concentration gradient are responsible for this material transport. ${ }^{43,44}$ The former gradient results from the temperature-dependence of surface tension, the temperature gradient given by the intensity profile of the beam and the heat capacity distribution in the sample. The latter results from the alteration of chemical composition due to the laser-induced intermixing between different layers (including the capping layer) and ab- or adsorption of other substances from the atmosphere (the laser irradiation was done in the normal atmosphere). A quantitative analysis of these material transport processes is quite complicated and beyond the scope of the present work. However in any case the observations done by AFM imply that there was melt flow during the laser irradiation, causing the intermixing between $\mathrm{Co}$ and Pd atoms where the extent of intermixing should be positively correlated with the laser intensity. Based 


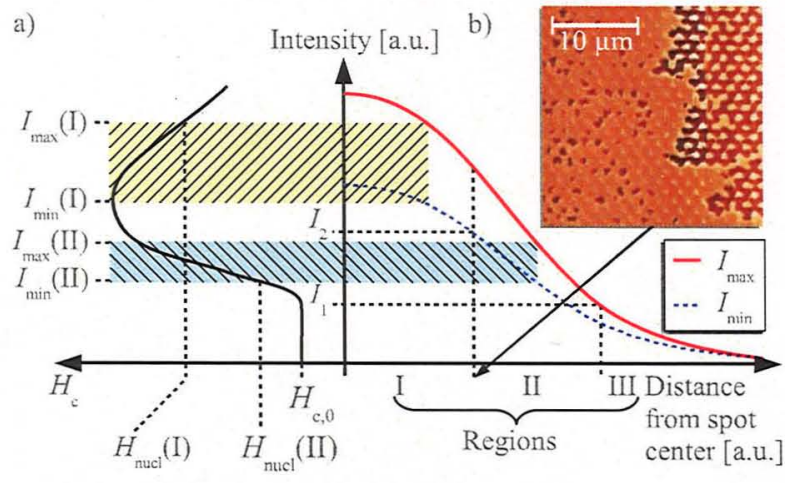

FIG. 9. (Color online) Schematic plots. (a) Coercivity $H_{c}$ of laser irradiated $\mathrm{Co} / \mathrm{Pd}$ multilayer vs laser intensity and (b) intensities of interference maxima and minima, $I_{\max }$ and $I_{\min }$, respectively vs distance from the center of the laser spot. The inset on the right shows an MFM micrograph of a transition region between regions I and II. $H_{c, 0}$ is the coercivity of the as-grown sample. The yellow and blue shaded areas represent the range of intensity with which 'a certain location within regions I and II, respectively, has been irradiated. $H_{\text {nucl }}(\mathrm{I})$ and $H_{\text {nucl }}(\mathrm{II})$ denote the smallest nucleation field at a certain location in regions I and II, respectively. $I_{1}$ and $I_{2}$ are the threshold intensities above which the laser irradiation starts to change $H_{c}$ and domain wall motion along the valleys is entirely inhibited, respectively.

on this idea, we now attempt to give a phenomenological explanation to the magnetization reversal processes observed here.

For this purpose, two schematic diagrams are shown in Fig. 9. The diagram in Fig. 9(a) illustrates the change in coercivity in $\mathrm{Co} / \mathrm{Pd}$ multilayers induced by laser irradiation. The diagram in Fig. 9(b) plots the beam intensity versus the distance from the center of the laser spot. The two Gaussian curves envelop the intensities of the interference maxima and minima. The latter is equal to zero only in the ideal case of perfect alignment of the beams, matching polarizations, and equality of intensities. Approaching the diagram in Fig. 9(b) from the far right side, one enters the rim of the irradiated area (region III) from the outer region, which remains unaltered from the as-grown state. As shown in Fig. 8, the laser intensity in region III is not high enough to cause any permanent changes, neither in the topography nor in the magnetic properties. This is indicated by Fig. 9(a) where it shows no laser-induced change in coercivity at low intensities. The intensity needs to be increased above a certain threshold $I_{1}$ so that the material can start to melt and flow, leading to structural changes in the multilayer system. This is where region II begins. The laser-induced material transport causes intermixing between the Co atoms and the Pd atoms at the interfaces, which increases the interfacial roughness. ${ }^{45}$ The sites with high interfacial roughness act as pinning centers obstructing domain growth and propagation. Since the extent of atomic intermixing at the interfaces grows with increasing laser intensity, the enhancement in coercivity is much greater at the interference maxima than at the minima as illustrated by blue shaded area in Fig. 9. This explains the switching behavior of region II shown in Figs. 6 and 7, where the magnetization in the valleys is reversed first and the switching of the hillocks occurs much later.

Moving closer to the center of the laser spot, the laser intensity traverses another threshold $I_{2}$ entering region $I$. The inset of Fig. 9 is an MFM micrograph, which shows a sharp boundary between region I (left) and region II (right). Beyond this boundary, on the side of region I, domain wall motion is entirely inhibited. Above $I_{2}$ the laser intensities must have been so high that even the valleys exhibit very high interfacial roughness and are also filled with pinning centers. As mentioned earlier, we have found in our previous studies $^{41}$ that the laser-induced chemical mixing of $\mathrm{Co}$ and $\mathrm{Pd}$ atoms at the interfaces not only increases the interfacial roughness but also reduces the strength of PMA. The weakening of the anisotropy lowers the domain wall energy, which in turn reduces the activation volume for domain nucleation. At some point this effect will outweigh the effect of pinning centers created by laser-induced roughening of interfaces, leading to the decrease in coercivity as illustrated in Fig. 9(a). Since the interference maxima reduce the anisotropy to a larger extent than the interference minima, the hillocks in region I switch first before the valleys, in contrast to the case of region II. The observations done in Figs. 4 and 5 confirm this.

\section{SUMMARY AND CONCLUSIONS}

We have used single-pulse DLIL to fabricate topographic as well as magnetic patterns in $\mathrm{Co} / \mathrm{Pd}$ multilayer systems where the intense laser heating at the interference maxima has altered the sample structure locally and generated a two-dimensional lattice. Due to its capability of patterning a large area, the DLIL produces a multitude of submicron-sized lattice elements within a very short period of time. This provides numerous samples for the investigations on the effect of highly localized laser irradiation. In addition, the inhomogeneous intensity distribution of the laser allows us to study the intensity-dependence of the patterning process and the resulting impact on the magnetic properties.

Using MFM, focused polar MOKE, and Kerr microscopy, we have found that the applied laser intensity has indeed significant influence on the magnetic properties of the patterned samples. The magnetic switching behavior differs substantially between the region irradiated at high intensities (region I) and the one irradiated at medium intensities (region II). The former is dominated by nucleation, while the latter by domain wall motion. Besides, the switching order of the patterns in region I is the exact opposite of that in region II. Compared to the publication by Aktag et al. ${ }^{18}$ who reported the formation of an anisotropy lattice of one single kind on Co/Pt multilayers, our studies show that for the $\mathrm{Co} / \mathrm{Pd}$ multilayers two different kinds of coercivity lattices are generated by laser irradiation at two different intensity ranges. At very low intensities, there is further a region (region III) exhibiting a different behavior where no permanent alteration is found to have occurred, neither to the topography nor to the magnetic properties. However, a volatile magnetic pattern is observed, which vanishes when an external magnetic field is applied.

We have developed a model that gives a phenomenological explanation for the observations made in these three regions. The model is based on the following considerations. 
First, the effects of laser irradiation of $\mathrm{Co} / \mathrm{Pd}$ multilayer system can be categorized into three types: (1) thermal magnetic excitation, (2) the creation of pinning centers by laserinduced roughening of interfaces, and (3) the reduction in anisotropy by intermixing between the Co and $\mathrm{Pd}$ atoms at the interfaces. Second, depending on the intensity range, one of these three processes becomes most pronounced, dominating the other two: process (1) is dominant in region III, process (2) in region II, and process (3) in region I.

To provide better understanding of the microscopic mechanisms beyond this phenomenological explanation, we are currently investigating the material dependence and formation dynamics of the topographical and structural alteration by DLIL in more detail.

\section{ACKNOWLEDGMENTS}

We gratefully acknowledge the financial support from the DFG granted to the work done at the University of Konstanz in the framework of Emmy Noether Programme and Collaborative Research Centre Programme SFB 513 (project A8) and to the Kerr microscopy studies carried out at the Ruhr-University Bochum in the framework of Collaborative Research Centre Programme SFB 491 (project N1).

${ }^{1}$ S. Y. Chou, M. S. Wei, P. R. Krauss, and P. B. Fischer, J. Appl. Phys. 76, 6673 (1994).

${ }^{2}$ G. Hu, T. Thomson, M. Albrecht, M. E. Best, B. D. Terris, C. T. Rettner, S. Raoux, G. M. McClelland, and M. W. Hart, J. Appl. Phys. 95, 7013 (2004)

${ }^{3}$ J. Lohau, A. Moser, C. T. Rettner, M. E. Best, and B. D. Terris, Appl. Phys. Lett. 78, 990 (2001)

${ }^{4}$ M. Albrecht, C. T. Rettner, M. E. Best, and B. D. Terris, Appl. Phys. Lett. 83, 4363 (2003).

${ }^{5}$ H. Tan, A. Gilbertson, and S. Y. Chou, J. Vac. Sci. Technol. B 16, 3926 (1998).

${ }^{6} \mathrm{C}$. Chappert, H. Bernas, J. Ferré, V. Kottler, J.-P. Jamet, Y. Chen, E. Cambril, T. Devolder, F. Rousseaux, V. Mathet, and H. Launois, Science 280, 1919 (1998).

${ }^{7}$ B. D. Terris, D. Weller, L. Folks, J. E. E. Baglin, A. J. Kellock, H. Rothuizen, and P. Vettiger, J. Appl. Phys. 87, 7004 (2000).

${ }^{8}$ M. Albrecht, G. Hu, I. L. Guhr, T. C. Ulbrich, J. Boneberg, P. Leiderer, and G. Schatz, Nature Mater. 4, 203 (2005)

${ }^{9}$ N. I. Polushkin, Appl. Phys. Lett. 86, 132502 (2005).

${ }^{10} \mathrm{C}$. Schuppler, A. Habenicht, I. L. Guhr, M. Maret, P. Leiderer, J. Boneberg, and M. Albrecht, Appl. Phys. Lett. 88, 012506 (2006).

${ }^{11}$ N. Y. Polushkin, S. A. Gusev, M. N. Drozdov, Y. K. Verevkin, and V. N. Petryakov, J. Appl. Phys. 81, 5478 (1997).

${ }^{12}$ A. E. Tselev, N. I. Polushkin, Y. K. Verevkin, A. A. Gorbunov, V. N. Petryakov, and W. Pompe, Appl. Phys. A: Mater. Sci. Process. 69, $\$ 819$ (1999).

${ }^{13}$ V. I. Bredikhin, Y. K. Verevkin, E. Y. Daume, S. P. Kuznetsov, O. A. Mal'shakova, V. N. Petryakov, N. V. Vostokov, and N. I. Polushkin, Quantum Electron. 30, 333 (2000).

${ }^{14}$ M. Zheng, M. Yu, Y. Liu, R. Skomski, S. H. Liou, D. J. Sellmyer, V. N.
Petryakov, Y. K. Verevkin, N. I. Polushkin, and N. N. Salashchenko, IEEE Trans. Magn. 37, 2070 (2001).

${ }^{15}$ M. Zheng, M. Yu, Y. Liu, R. Skomski, S. H. Liou, D. J. Sellmyer, V. N. Petryakov, Y. K. Verevkin, N. I. Polushkin, and N. N. Salashchenko, Appl. Phys. Lett. 79, 2606 (2001).

${ }^{16}$ L. Gao, S. H. Liou, M. Zheng, R. Skomski, M. L. Yan, D. J. Sellmyer, and N. I. Polushkin, J. Appl. Phys. 91, 7311 (2002).

${ }^{17}$ N. I. Polushkin, J. Wittborn, C. Canalias, K. V. Rao, A. M. Alexeev, and A. F. Popkov, J. Appl. Phys. 92, 2779 (2002).

${ }^{18}$ A. Aktag, S. Michalski, L. Yue, R. D. Kirby, and S.-H. Liou, J. Appl. Phys. 99, 093901 (2006)

${ }^{19}$ Magnetic Heterostructures, Springer Tracts in Modern Physics Vol. 227, edited by H. Zabel and S. D. Bader (Springer-Verlag, Berlin, Heidelberg, 2008).

${ }^{20}$ T. Suzuki, Z. Zhang, A. Singh, J. Yin, A. Perumal, and H. Osawa, IEEE Trans. Magn. 41, 555 (2005)

${ }^{21}$ S. Mangin, D. Ravelosona, J. A. Katine, M. J. Carey, B. D. Terris, and E. E. Fullerton, Nature Mater. 5, 210 (2006).

${ }^{22}$ S. N. Piramanayagam, J. Appl. Phys. 102, 011301 (2007).

${ }^{23}$ J.-H. Park, C. Park, T. Jeong, M. T. Moneck, N. T. Nufer, and J.-G. Zhu, J. Appl. Phys. 103, 07A917 (2008).

${ }^{24}$ P. F. Carcia, A. D. Meinhaldt, and A. Suna, Appl. Phys. Lett. 47, 178 (1985).

${ }^{25}$ F. J. A. den Broeder, H. C. Donkersloot, H. J. G. Draaisma, and W. J. M. de Jonge, J. Appl. Phys. 61, 4317 (1987).

${ }^{26}$ P. F. Carcia, J. Appl. Phys. 63, $5066(1988)$

${ }^{27}$ F. J. A. den Broeder, W. Hoving, and P. J. H. Bloemen, J. Magn. Magn. Mater. 93, 562 (1991).

${ }^{28}$ D. Weller, Y. Wu, J. Stöhr, M. G. Samant, B. D. Hermsmeier, and C. Chappert, Phys. Rev. B 49, 12888 (1994).

${ }^{29} \mathrm{C}$. Chappert and P. Bruno, J. Appl. Phys. 64, 5736 (1988).

${ }^{30}$ J. Carrey, A. E. Berkowitz, W. F. Egelhoff, Jr., and D. J. Smith, Appl. Phys. Lett. 83, 5259 (2003).

${ }^{31}$ J. I. Hong, S. Sankar, A. E. Berkowitz, and W. F. Egelhoff, Jr., J. Magn. Magn. Mater. 285, 359 (2005).

${ }^{32}$ Y. Wu, J. Stöhr, B. D. Hermsmeier, M. G. Samant, and D. Weller, Phys. Rev. Lett. 69, 2307 (1992).

${ }^{33}$ Y. K. Schuller, S. Kim, and C. Leighton, J. Magn. Magn. Mater. 200, 571 (1999).

${ }^{34}$ D. Weller, J. E. E. Baglin, A. J. Kellock, K. A. Hannibal, M. F. Toney, G. Kusinski, S. Lang, L. Folks, M. E. Best, and B. D. Terris, J. Appl. Phys. 87, $5768(2000)$.

${ }^{35} \mathrm{~J}$. Fassbender, D. Ravelosona, and Y. Samson, J. Phys. D 37, R179 (2004).

${ }^{36}$ D. Stanescu, D. Ravelosona, V. Mathet, C. Chappert, Y. Samson, C. Beigné, N. Vernier, J. Ferré, J. Gierak, E. Bouhris, and E. E. Fullerton, J. Appl. Phys. 103, 07B529 (2008).

${ }^{37}$ H. J. G. Draaisma and W. J. M. de Jonge, J. Appl. Phys. 62, 3318 (1987).

${ }^{38}$ J. R. Barnes, S. J. O'Shea, M. E. Welland, J.-Y. Kim, J. E. Evetts, and R. E. Somekh, J. Appl. Phys. 76, 2974 (1994).

${ }^{39}$ S.-B. Choe and S.-C. Shin, Phys. Rev. B 57, 1085 (1998).

${ }^{40}$ S.-B. Choe and S.-C. Shin, Phys. Rev. B 59, 142 (1999).

${ }^{41}$ P. M. Leufke, Diploma thesis, University of Konstanz, 2007.

${ }^{42}$ A. M. Alekseev, Y. K. Verevkin, N. V. Vostokov, V. N. Petryakov, N. I. Polushkin, A. F. Popkov, and N. N. Salashchenko, JETP Lett. 73, 192 (2001).

${ }^{43}$ D. Bäuerle, Laser Processing and Chemistry, 2nd ed. (Springer-Verlag, Berlin, Heidelberg, 1996).

${ }^{44} \mathrm{~S}$. Riedel, Diploma thesis, University of Konstanz, 2007.

${ }^{45}$ T. Suzuki, H. Notarys, D. C. Dobbertin, C.-J. Lin, D. Weller, D. C. Miller, and G. Gorman, IEEE Trans. Magn. 28, 2754 (1992). 